\title{
Surgical risk models: how they contribute to improvement of our treatments?
}

\author{
Janusz R. Włodarczyk, Jarosław Kużdżał \\ Department of Thoracic and Surgical Oncology, Jagiellonian University Collegium Medicum, John Paul II Hospital, Cracow, Poland \\ Correspondence to: Janusz R. Włodarczyk, MD, PhD. Department of Thoracic Surgery, John Paul II Hospital, ul. Pradnicka 80, Cracow 31-202, \\ Poland. Email: jr.wlodarczyk@gmail.com. \\ Provenance: This is an Invited Editorial commissioned by Section Editor Dr. Min Zhang (Department of Thoracic Oncology, The First Affiliated \\ Hospital of Chongqing Medical University, Chongqing, China). \\ Comment on: Brunelli A, Salati M, Rocco G, et al. European risk models for morbidity (EuroLung1) and mortality (EuroLung2) to predict outcome \\ following anatomic lung resections: an analysis from the European Society of Thoracic Surgeons database. Eur J Cardiothorac Surg 2017;51:490-7.
}

Submitted Sep 19, 2017. Accepted for publication Oct 05, 2017.

doi: $10.21037 /$ jtd.2017.10.85

View this article at: http://dx.doi.org/10.21037/jtd.2017.10.85

Adverse events are common among surgical patients. The question of how to predict risk associated with invasive procedures and how to select an optimal procedure for particular patients is as old as surgery itself. Accurate calculation of complication risk and mortality risk has three important aspects:

(I) Risk assessment and its discussion with a patient is essential for their informed consent to undergo surgery;

(II) Determination of risk allows for implementation of specific measures, aimed at reduction of this risk;

(III) Awareness of the adverse event that is likely to happen enables one to get prepared for it, to provide optimal setting for the procedure and to use appropriate treatment without delay.

Numerous risk factors for different adverse events have been described and surgeons are well familiar with them. However, precise calculation of risk in the actual patient is not a simple task. This is because the multifactorial nature of complications' aetiology, and very complex interplay between these factors and intrinsic patient's characteristics. Also, for reliable statistical analysis, reliable data are necessary.

Development of appropriate databases is extremely difficult, time-consuming and an expensive task. Ideally, such a database should include prospectively collected sets of relevant data, collected in a non-selective way in a large cohort of patients operated on over a relatively short period of time. In fact, this means multi-institutional and even international projects, run by highly professional teams including surgeons, IT specialists and statisticians, supported by a large number of high-volume institutions providing patients' data.

There is no doubt that such an ideal database does not exist, due to the abovementioned inherent problems. There are, however, databases, which were used to produce risk models that are successfully used by thoracic surgeons worldwide. The first of them is the Thoracoscore, derived from the national French database, Epithor (1). The second to be mention is the database of the Society of Thoracic Surgeons (2). The most recent attempt to create improved risk model was presented during the conference of the European Society of Thoracic Surgeons (ESTS) in Naples in 2016, and published thereafter in the European Journal of Cardiothoracic Surgery (3).

This latter risk model is particularly promising because the ESTS database meets several of the criteria of an ideal surgical database: it is collected prospectively, using uniform, web-based tool, it contains most of the important clinical information and includes data from multiple institutions collected in a short period of time. The authors used data of 47,960 patients operated on between 2007 and 2015. Using advanced statistical methods, they developed two risk models: for morbidity (EuroLung1) and mortality (EuroLung2).

The bootstrap method, introduced in the1970s by Efron, is becoming more and more popular due to availability of computers of high computing power and sophisticated 
software (4). It is generally based on creation of artificial population by resampling of the original, finite sample.

Brunelli et al. have used 8 variables to create the EuroLung1 and 9 variables for EuroLung2. The variables found to be associated with morbidity after logistic regression analysis include: gender, age, predicted postoperative FEV1, coronary artery disease, cerebrovascular disease, renal failure, thoracotomy approach and extended resections. The aggregate EuroLung1 stratifies the patients into 6 categories of incremental risk of complications. On the other hand, the variables found to be associated with mortality after logistic regression analysis included: gender, age, predicted postoperative FEV1, coronary artery disease, cerebrovascular disease, BMI, thoracotomy approach, pneumonectomy and extended resections. The aggregate EuroLung2 score stratifies patients into 6 categories of incremental risk of mortality.

The new risk models have some limitations. Firstly, the ESTS database does not include all pulmonary resections. Approximately $20 \%$ of European thoracic surgical departments contribute to the database. Moreover, as contribution is voluntary, there is a bias due to a larger representation of high-volume, university-based centres. Some data, like DLCO or $\mathrm{VO}_{\max }$ were available for a minority of patients and were therefore not included in the final analysis. Mortality was reported for the 30-day period, so the 90-day mortality in unknown. Also, the category 'thoracotomy approach' that found a risk factor for both, morbidity and mortality, does not differentiate between large postero-lateral approach and the muscle-sparing mini-thoracotomy; this is likely to be the reason for bias regarding this parameter.

Nevertheless, the EuroLung1 and EuroLung2 models are probably the most reliable risk models available today. They present important progress in our struggle to preoperatively define surgical risk and, therefore, to improve results of treatment of patients with lung cancer.

Regrettably, there is no web-based, simple calculator available, that could be used to calculate the EuroLung1 and EuroLung2 scores, as it was done for the Thoracoscore. Let us hope that such calculator will appear soon on the web page of ESTS.

\section{Acknowledgements}

None.

\section{Footnote}

Conflicts of Interest: The authors have no conflicts of interest to declare.

\section{References}

1. Bernard A, Rivera C, Pages PB, et al. Risk model of inhospital mortality after pulmonary resection for cancer: a national database of the French Society of Thoracic and Cardiovascular Surgery (Epithor). J Thorac Cardiovasc Surg 2011;141:449-58.

2. Fernandez FG, Kosinski AS, Burfeind W, et al. The Society of Thoracic Surgeons Lung Cancer Resection Risk Model: Higher Quality Data and Superior Outcomes. Ann Thorac Surg 2016;102:370-7.

3. Brunelli A, Salati M, Rocco G, et al. European risk models for morbidity (EuroLung1) and mortality (EuroLung2) to predict outcome following anatomic lung resections: an analysis from the European Society of Thoracic Surgeons database. Eur J Cardiothorac Surg 2017;51:490-7.

4. Efron B. Boostrap Methods: Another Look at the Jackknife. Ann Stat 1979;7:1-26.
Cite this article as: Włodarczyk JR, Kużdżał J. Surgical risk models: how they contribute to improvement of our treatments? J Thorac Dis 2017;9(11):4195-4196. doi: 10.21037/ jtd.2017.10.85 